\title{
A Model for Shear Layer Effects on Engine Noise Radiation
}

\author{
Douglas M. Nark, F. Farassat, D. Stuart Pope, and V. Vatsa ${ }^{\S}$ \\ NASA Langley Research Center, Hampton, Virginia
}

\begin{abstract}
Prediction of aircraft engine noise is an important aspect of addressing the issues of community noise and cabin noise control. The development of physics based methodologies for performing such predictions has been a focus of Computational Aeroacoustics (CAA). A recent example of code development in this area is the ducted fan noise propagation and radiation code CDUCT-LaRC. Included within the code is a duct radiation model that is based on the solution of Ffowcs Williams-Hawkings (FW-H) equation with a penetrable data surface. Testing of this equation for many acoustic problems has shown it to provide generally better results than the Kirchhoff formula for moving surfaces. Currently, the data surface is taken to be the inlet or exhaust plane for inlet or aft-fan cases, respectively. While this provides reasonable results in many situations, these choices of data surface location lead to a few limitations. For example, the shear layer between the bypass flow and external stream can refract the sound waves radiated to the farfield. Radiation results can be improved by including this effect, as well as the reflection of the sound in the bypass region from the solid surface external to the bypass duct surrounding the core flow. This work describes the implementation, and possible approximation, of a shear layer boundary condition within CDUCT-LaRC. An example application also illustrates the improvements that this extension offers for predicting noise radiation from complex inlet and bypass duct geometries, thereby providing a means to evaluate external treatments in the vicinity of the bypass duct exhaust plane.
\end{abstract}

${ }^{*}$ Research Scientist, Structural Acoustics Branch, Aerodynamics, Aerothermodynamics, and Acoustics Competency, AIAA Member

†Senior Research Scientist, Aeroacoustics Branch, Aerodynamics, Aerothermodynamics, and Acoustics Competency, AIAA Associate Fellow

$\ddagger$ Analytical Services and Materials

${ }^{\S}$ Senior Research Scientist, Computational Modeling and Simulation Branch, Aerodynamics, Aerothermodynamics, and Acoustics Competency 


\section{Introduction}

Prediction of aircraft engine noise is an important aspect of addressing the issues of community noise and cabin noise control. The development of physics based methodologies for performing such predictions has been a focus of Computational Aeroacoustics (CAA). A recent example of code development within this area is the ducted fan noise propagation and radiation code CDUCT-LaRC. ${ }^{1}$ Included within the code is an acoustic radiation model that is based on the Ffowcs Williams-Hawkings (FW-H) equation with a penetrable data surface. ${ }^{2}$ Testing of this equation for many acoustic problems has shown it to generally provide better results than the Kirchhoff formula for moving surfaces. Currently within CDUCT-LaRC, the data surface for radiation calculations is taken to be the inlet or exhaust plane for inlet or aft-fan cases, respectively. While this provides excellent results in many situations, these data surface locations may lead to a few limitations. The first involves the effects of the shear layer on acoustic radiation. With the data surface at the exhaust plane, refraction of sound through the shear layer may not be taken into account. Secondly, difficulties may arise when large aft or forward radiation angles are of interest for inlet or aft-fan cases, respectively. In these situations, the effects of the engine cowl are not taken into account and the acoustic results are essentially symmetric about the data surface.

The discussion to follow describes one approach to alleviating these issues within the CDUCTLaRC framework by extending the propagation calculations beyond the exhaust plane of the bypass duct. An impedance boundary condition to be applied on the surface of the shear layer is derived. This is followed by the implementation of this boundary condition and possible areas for approximation. Preliminary results are then presented to illustrate some initial examples utilizing the extended propagation calculations. Finally, concluding remarks are offered, as well as areas for further development and applicability.

\section{Derivation}

The shear layer between the bypass flow and external stream can refract the sound waves radiated to the farfield. Radiation results can be improved by including this effect, as well as the reflection of the sound in the bypass region from the solid surface external to the bypass duct surrounding the core flow (see figure 1). One way to achieve this is to extend the CDUCT-LaRC bypass duct propagation calculation, which is based on the parabolic approximation, beyond the exhaust plane and to apply a boundary condition (BC) similar to a liner BC on the shear layer.

Although this derivation has been presented in reference, ${ }^{1}$ it is useful to revisit the details in this work. The development of this boundary condition begins with the satisfaction of two conditions on the shear layer:

1. The particle displacement must be continuous across the shear layer.

2. The acoustic pressure must be continuous across the shear layer. 


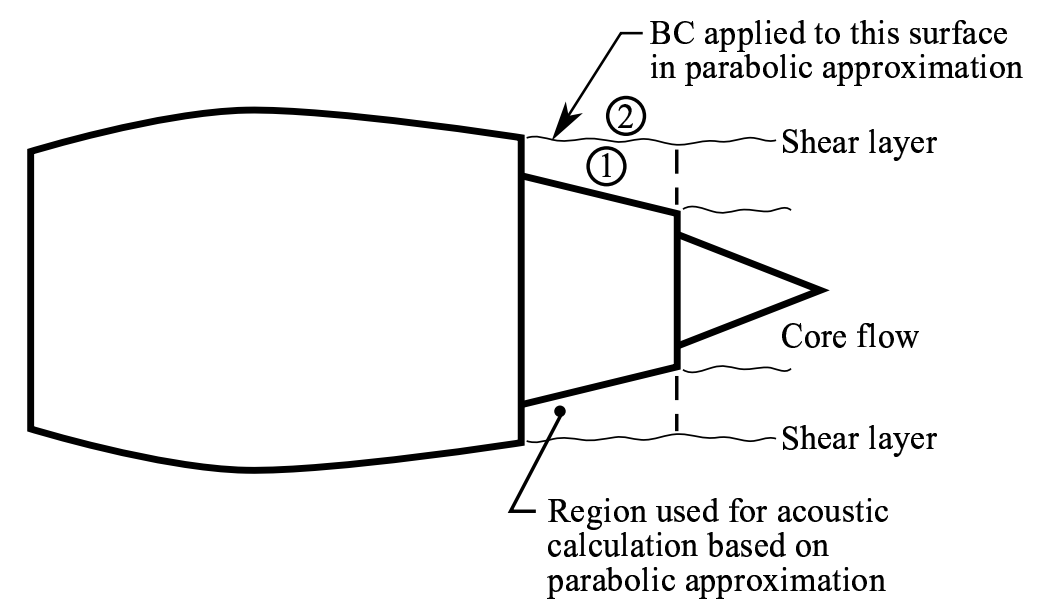

Figure 1. Boundary Regions for the Aft-Fan Geometry

The first result involves the acoustic velocity normal to the shear layer. This follows a derivation of the liner BC by Myers ${ }^{3}$ as presented by Farassat and Dunn. ${ }^{4}$ The subscripts 0 and 1 are used for the background and acoustic quantities. The particle displacement amplitude normal to the shear layer is also denoted by $q_{3}$ and the time dependence is harmonic with the factor $e^{-i \omega t}$. Following the notation of Farassat and Dunn ${ }^{4}$ [eq. (3) with $\varepsilon=1$ ], one may write

$$
\begin{aligned}
\frac{\partial q_{3}}{\partial t}=-i \omega q_{3} & =\left[q_{3} \overrightarrow{n_{0}} \cdot\left(\overrightarrow{n_{0}} \cdot \nabla \overrightarrow{u_{0}}\right)+\overrightarrow{u_{1}} \cdot \overrightarrow{n_{0}}\right]^{1} \\
& =\left[q_{3} \overrightarrow{n_{0}} \cdot\left(\overrightarrow{n_{0}} \cdot \nabla \overrightarrow{u_{0}}\right)+\overrightarrow{u_{1}} \cdot \overrightarrow{n_{0}}\right]^{2}
\end{aligned}
$$

where the superscripts 1 and 2 designate regions 1 and 2 in figure $1, \vec{u}$ is the fluid velocity, and $\vec{n}$ is the unit normal to the shear layer.

From equation 1, one finds

$$
q_{3}=\frac{-u_{1 n}^{1}}{i \omega+\left[\overrightarrow{n_{0}} \cdot\left(\overrightarrow{n_{0}} \cdot \nabla \overrightarrow{u_{0}}\right)\right]^{1}}=\frac{-u_{1 n}^{2}}{i \omega+\left[\overrightarrow{n_{0}} \cdot\left(\overrightarrow{n_{0}} \cdot \nabla \overrightarrow{u_{0}}\right)\right]^{2}} .
$$

and

$$
u_{1 n}^{2}=\frac{i \omega+\left[\overrightarrow{n_{0}} \cdot\left(\overrightarrow{n_{0}} \cdot \nabla \overrightarrow{u_{0}}\right)\right]^{2}}{i \omega+\left[\overrightarrow{n_{0}} \cdot\left(\overrightarrow{n_{0}} \cdot \nabla \overrightarrow{u_{0}}\right)\right]^{1}} u_{1 n}^{1} .
$$

This result shows that if $u_{1 n}^{1}$ is found on the shear layer from the parabolic approximation method in region 1 , then $u_{1 n}^{2}$ on the external side of the shear layer may be calculated.

Next, the derivation of the impedance, $z_{2}$, on the side of the shear layer adjacent to region 2 is presented beginning with the model and assumptions. ${ }^{\text {a }}$ First, the shear layer is taken to be infinitely

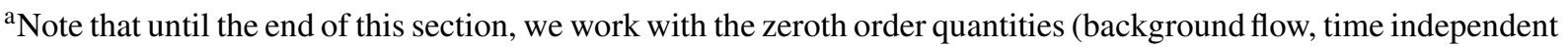
quantities). For simplicity of notation, we denote regions 1 and 2 by subscripts. 
thin, an assumption previously utilized in equation 3. Second, the barber pole pattern of the modes present in the flow region is assumed to persist in the flow region inside the shear layer. This barber pole pattern has a trace velocity on the shear layer surface in the direction normal to itself. Figure 2 shows this trace velocity, $\vec{V}_{s}$, and the method in which it is calculated. This figure also shows a local tangent plane to the shear layer surface which is cylindrical. Assuming the acoustic

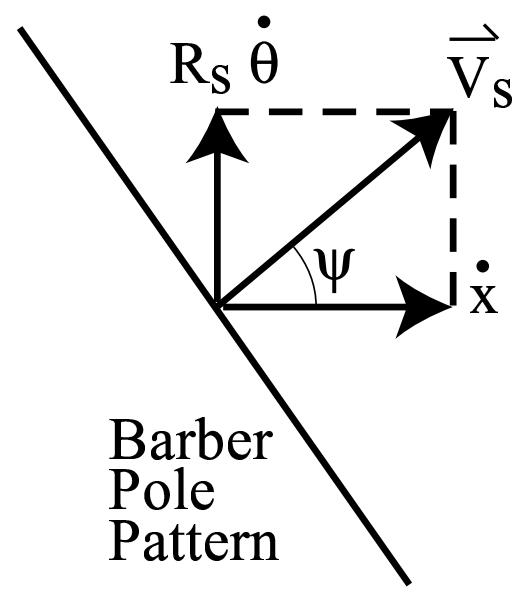

Figure 2. Trace Velocity Description

pressure in a mode to be proportional to $\exp \left[-i\left(\omega t-m \theta-k_{x} x\right)\right]$ where $m$ is the circumferential mode number, $x$ is the axial distance, and $k_{x}$ is the axial wave number, it follows that

$$
\begin{gathered}
\dot{\theta}=\frac{\omega}{m}, \dot{x}=\frac{\omega}{k_{x}} \\
R_{s} \dot{\theta}=\frac{\omega / c_{1}}{m} R_{s} c_{1}=\frac{k R_{s}}{m} c_{1} \\
\dot{x}=\frac{\omega / c_{1}}{k_{x}} c_{1}=\frac{k}{k_{x}} c_{1} \\
V_{s}=\left\|\vec{V}_{s}\right\|=k c_{1} \sqrt{\left(\frac{R_{s}}{m}\right)^{2}+\frac{1}{k_{x}^{2}}} .
\end{gathered}
$$

Here, $R_{s}$ is the local radius of the shear layer (assumed almost constant), $k=\omega / c_{1}$, and $c_{1}$ is the local speed of sound in region 1 of the shear layer. Noting that this expression relates to $m \neq 0$, the zero order circumferential mode may also be considered with little difficulty. Here, the acoustic pressure in a mode is proportional to $\exp \left[-i\left(\omega t-k_{x} x\right)\right]$. Therefore,

$$
\dot{\theta}=0, \dot{x}=\frac{\omega}{k_{x}}
$$




$$
V_{s}=\left\|\overrightarrow{V_{s}}\right\|=\frac{k c_{1}}{k_{x}}
$$

Additionally, the trace velocity for the plane wave case may be simplified further. Since the axial wave number for the right traveling plane wave is written $k_{x}=\frac{k}{1+M}$, it follows that the trace velocity is independent of frequency and is given by

$$
V_{s}=c_{1}(1+M) \text {. }
$$

From the un-numbered equation above equation 11.1.21 in Morse and Ingard, ${ }^{5}$ the impedance, $z_{2}$, is

$$
z_{2}=\frac{\rho_{2} c_{2}}{\left(1+M_{2} \cos \phi\right) \sin \phi}
$$

where $M_{2}=u_{2} / c_{2}$ is the Mach number based on the background flow in region 2 and $\phi$ is the preferred direction of propagation of plane waves in region 2. This angle is found purely by kinematic considerations. Figure 3 shows the geometry in the local plane containing $\vec{V}_{s}$ and the local normal to the shear layer which is along axis 2 . Let $\vec{e}=(\sin \phi, \cos \phi)$ be the direction normal to the wavefront. The effective wave speed in region $2, \overrightarrow{c_{e}}$, is

$$
\overrightarrow{c_{e}}=c_{2} \vec{e}+\overrightarrow{u_{2}} \cos \psi
$$

where $\cos \psi=\dot{x} / V_{s}$ is shown in figure 2 . The projection of $\overrightarrow{c_{e}}$ on the shear layer is equal to the

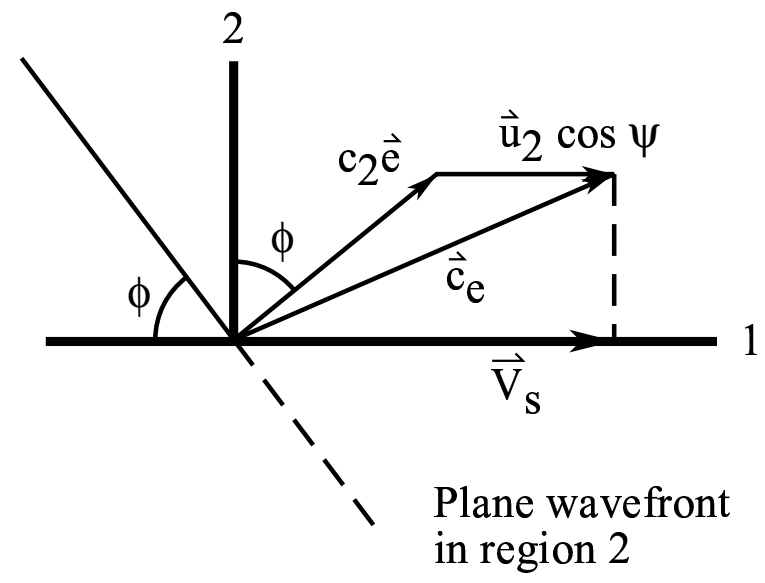

Figure 3. Effective Wave Speed Description

trace speed $V_{s}$. This gives

$$
V_{s}=u_{2} \cos \psi+c_{2} \sin \phi
$$


from which it is seen that

$$
\sin \phi=\frac{V_{s}-u_{2} \cos \psi}{c_{2}}
$$

Using this in equation 11 leads to (equation 11.1.12 of Morse and Ingard ${ }^{5}$ )

$$
z_{2}=\frac{\rho_{2} c_{2}\left(V_{s}-u_{2}\right)^{2}}{V_{s} \sqrt{\left(V_{s}-u_{2}\right)^{2}-c_{2}^{2}}} .
$$

The final step in the formulation is that

$$
z_{1}=\frac{u_{1 n}^{2}}{u_{1 n}^{1}} z_{2}
$$

and equation 3 provides an expression for $u_{1 n}^{2} / u_{2 n}^{1}$.

\section{Implementation}

Implementation of the aforementioned impedance boundary condition rests on the calculation of two main quantities. The first is the ratio of particle velocity external to the shear layer (region 2) to the particle velocity internal to the shear layer (region 1), $u_{1 n}^{2} / u_{2 n}^{1}$. The second is the impedance on the external side of the shear layer, $z_{2}$. Determination of the ratio of particle velocities, (eq. 3), requires knowledge of the background flow velocity vectors and the unit normal to the shear layer surface. The background flow velocity vectors are available from the steady compressible flow solution obtained within the background flow module of CDUCT-LaRC. Additionally, the location of the shear layer may be obtained from the background flow solution. The Non-Uniform Rational B-Splines (NURBS) techniques utilized within the grid generation and redistribution functionality of CDUCT-LaRC then provide the ability to define the shear layer surface and therefore, the unit normal to the shear layer. Coincidentally, the NURBS functionality also provides a means for using the background flow grid and solution to create an acoustic grid suitable for propagation calculations (i.e. as orthogonal and uniform as possible).

Knowledge of the shear layer surface also extends to the calculation of the impedance on the external side of the shear layer, as it depends on the local shear layer radius. However, as seen in equation 14 , calculation of $z_{2}$ also requires knowledge of the circumferential mode number, $m$, and the axial wave number, $k_{x}$. When the background flow in region 1 of the shear layer is uniform, this presents little problem, as the propagation solution for the internal portions of the duct may be decomposed into duct modes and the 'important' modes may be treated individually. Assuming that a hardwall section exists at the exhaust plane. However, this approach may lead to difficulties when the background flow is non-uniform, as the modal decomposition may not be well defined. In these situations, it is possible that straight-forward decomposition into fundamental annular duct modes may lead to non-physical modal amplitudes as the radial mode number is increased beyond those that should be cut-off. It would therefore seem beneficial to attempt an approximation to the external impedance, $z_{2}$, that is independent of radial mode number. 
As the accuracy of the parabolic approximation decreases for modes nearing cut-off, a reasonable approach may be to use the axial wave number of the first radial mode. Thus, for a given circumferential mode, the axial wave number for the first radial mode would be used in equations 7 and 9 to obtain the trace velocity for all cut-on modes of a given circumferential order. It should be noted that this approximation does not lead to impedance values, $z_{2}$, that are independent of frequency, as in the plane wave case. On the contrary, the axial wave number generally is a function of frequency and this approach results in frequency dependent impedance values.

To explore the effects of this approximation while reducing the effects of other factors, consider a straight annular geometry with uniform flow in the internal (region 1) and external (region 2) areas of the shear layer. This alleviates the effects of a non-unity ratio of particle velocity (eq. 3 ). In addition, the radius of the shear layer, $R_{s}$, may then be taken to be constant so that the trace velocity changes only with circumferential mode, $m$, and axial wave number, $k_{x}$. Initially, take the duct to have dimensions similar to a business jet geometry with inner and outer radii of $0.303 \mathrm{~m}$ (11.9 in) and $0.391 \mathrm{~m}$ (15.4 in), respectively. With $M_{1}=0.5$ and $M_{2}=0.3$, the normalized (with respect to $\rho c$ ) shear layer resistance, $z_{2}$, for all cut-on radial modes are shown for $m=1,2,5,7$, and 10 in figure 4. The results for the zero order circumferential mode are not included as only one mode, other than the plane wave, is cut-on.

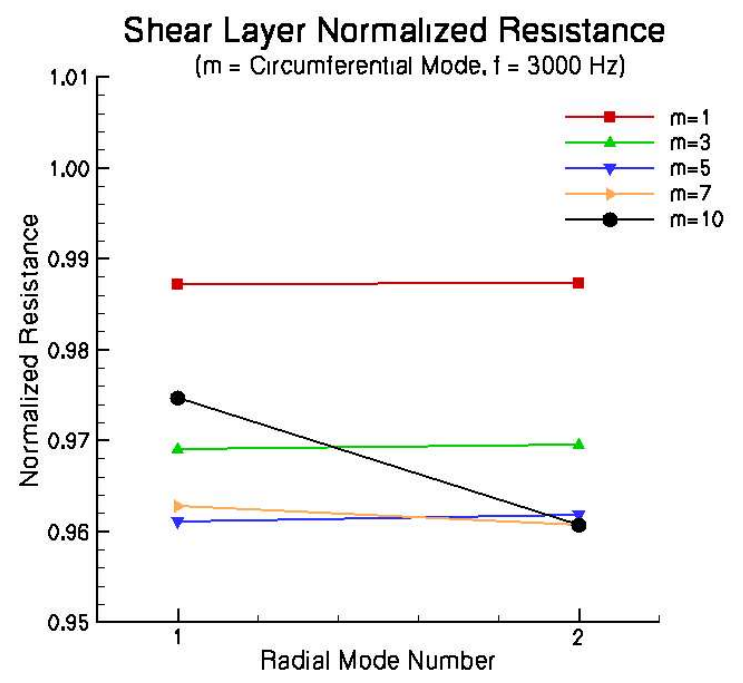

Figure 4. Shear Layer Resistance for $m=1,2,5,7$, and 10

It should be noted that only the resistance is presented because the reactance is zero based on the given flow assumptions. Equation 14 shows that in order for $z_{2}$ to be imaginary, $v_{s}-u_{2}<c_{2}$, which is not satisfied by the conditions specified above. Additionally, the possibility of non-zero reactance that is introduced through equation 3 is also suppressed by the specified flow conditions. In this case, as circumferential mode number increases, it appears that the resistance tends to decrease slightly with increasing radial mode number. However, it can be seen that the calculated 
resistance values are nearly constant for the lower order radial modes. Thus, for these conditions, it appears that using the axial wave number of the first radial mode in equation 7 for subsequent radial modes may be a reasonable assumption. For example, the $(10,1)$ has a cut-off ratio of 1.8 for this geometry and flow conditions, one could therefore hope that treatment within the bypass duct would attenuate this mode so that it's importance is diminished near the exhaust plane.

Carrying the analysis further, another annular geometry with dimensions similar to a larger commercial engine (inner and outer radii of $1.17 \mathrm{~m}$ (46.1 in) and $2.26 \mathrm{~m}$ (89 in), respectively) may be considered. With $M_{1}=0.7$ and $M_{2}=0.3$, the shear layer impedance for $m=1,2,5,7$, and 10 are shown in figure 5 at a source frequency of $1600 \mathrm{~Hz}$.

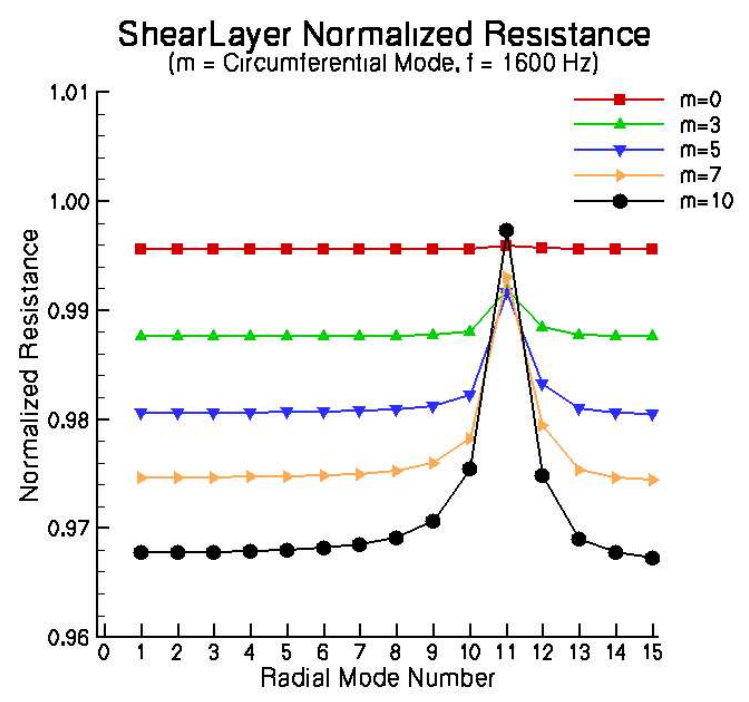

Figure 5. Shear Layer Resistance for $m=1,2,5,7$, and 10

In this case, a much wider range of radial modes are cut-on and it can be seen that the shear layer resistance remains fairly constant for the lower order radial modes (i.e. 1 9) . As with the previous geometry, this is less accurate for higher order circumferential modes which appears to be due to the fact that the $\frac{R_{s}}{m}$ term in the trace velocity decreases for larger $m$. Similarity to the previous case aside, there is another feature to notice in the resistance plot. As the radial mode number increases past 9, there is a large increase in resistance and then corresponding decrease back to the levels for the order modes. This peak in the values is associated with modes for which the axial wave number actually becomes negative and continues to grow negatively until cut-off. As discussed by Eversman, ${ }^{6}$ negative phase velocities may arise for wave propagation solutions in the presence of the mean flow. In such a situation, the applicable downstream solution has the smallest absolute value of $k_{x}$ with downstream power transmission. In these cases, the formulation of the trace velocity, and therefore impedance expression, may fall into question and bring about a possible area of further research. However, the importance of these modes may also be questioned, as again these are nearing cut-off and it is hoped that internal bypass duct treatment may attenuate 
them considerably. In addition, recall that as modes near cut-off, the accuracy of the parabolic approximation diminishes. It would therefore seem reasonable to verify that lower order modes (larger cut-off ratio) are properly treated at the expense of modes close to cut-off.

For this geometry, the zero order circumferential mode also has a wider range of radial modes cut-on. The impedance results for $m=0$ are shown in figure 6 . The resistance for the plane wave $(m=0, n=0)$ is not included as it may be treated separately and maintains a constant value over frequency given by equation 10. Again, as seen previously, the resistance appears to decrease as

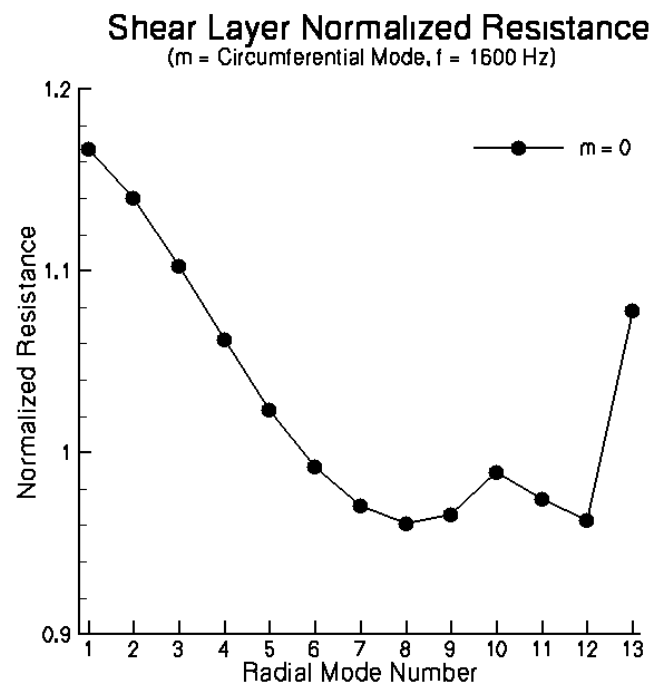

Figure 6. Shear Layer Resistance for $m=0$

radial mode number increases. However, in contrast to non-zero circumferential modes, it appears that the zero circumferential order modes does not maintain the nearly constant resistance values for the lower order radial modes. This appears to be due to the absence of the $\left(\frac{R_{s}}{m}\right)^{2}$ in the trace velocity (eq. 7) which is generally larger in magnitude than the term $\left(\frac{1}{k_{x}}\right)^{2}$. The trace velocity is therefore affected by a change in axial wave number and varies more noticeably with increasing radial mode number. It should also be noted that the resistance value for the $\mathrm{n}=14$ radial mode was not included in 6 . This is due to the fact that while this mode is barely cut-on, $V_{s}-u_{2}$ is less than $c_{2}$ and $z_{2}$ is purely imaginary (i.e. reactive). As discussed for the $m \neq 0$ cases in figure 5, the axial wave number for this mode is negative and the validity of equation 7 must be assessed. Based on these results, it may be the zero order circumferential modes, other than the plane wave, that are most effected by the axial wave number assumption presented. However, as mentioned previously, it is hoped that the importance of the higher order modes is diminished near the exhaust plane of the bypass duct by carrying less energy than the lower order modes and being attenuated more effectively by internal treatment. 


\section{Example Calculation}

Finally, a sample calculation for a more realistic geometry with a pylon extending beyond the exhaust plane of the bypass duct (figure 7) is briefly discussed. The geometry is taken to be that of the Jet Engine Simulator (JES) in the Jet Noise Laboratory of the NASA Langley Research Center.

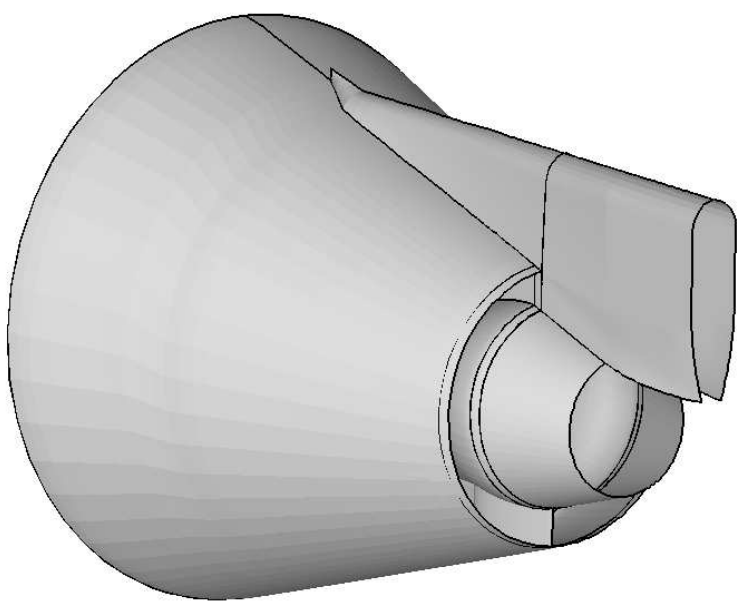

Figure 7. Jet Engine Simulator (JES) Geometry

As this is intended to illustrate a possible application of the shear layer boundary condition, simplified flow conditions are prescribed. The external Mach number, $M_{2}$, is set to a uniform value of 0.3. The Mach number internal to the shear layer is taken to obey 1D Mach/area relations with an initial Mach number of 0.7. The mean flow module of CDUCT-LaRC was used to approximate the shear layer surface location using inflow conditions set to match the Mach numbers mentioned above. This resulted in a pseudo duct geometry as shown in figure 8 . This is essentially a duct extending from the exhaust plane of the bypass duct to the trailing edge of the core cowl with the inner and outer walls composed of the core cowl and shear layer surfaces, respectively. Additionally, the duct is split due to the presence of pylon, shown as solid surfaces (shaded) in figure 8 .

Typically, the background flow solution would also be used to determine local Mach number values for the determination of the shear layer impedance and 'pseudo-duct' propagation calculations. However, since this is an illustrative example, the simplified mean flow conditions were used. Assuming a plane wave source at $1600 \mathrm{~Hz}$, the shear layer boundary condition was applied and contours of the real part of acoustic potential are presented in figure 9. These contours are shown in relation to the entire geometry to further illustrate the location of the propagation calculations.

Radiation calculations may then be performed within the CDUCT-LaRC framework using the exposed (outer) surfaces of the pseudo-duct as source surfaces. Treatments on any of the solid 


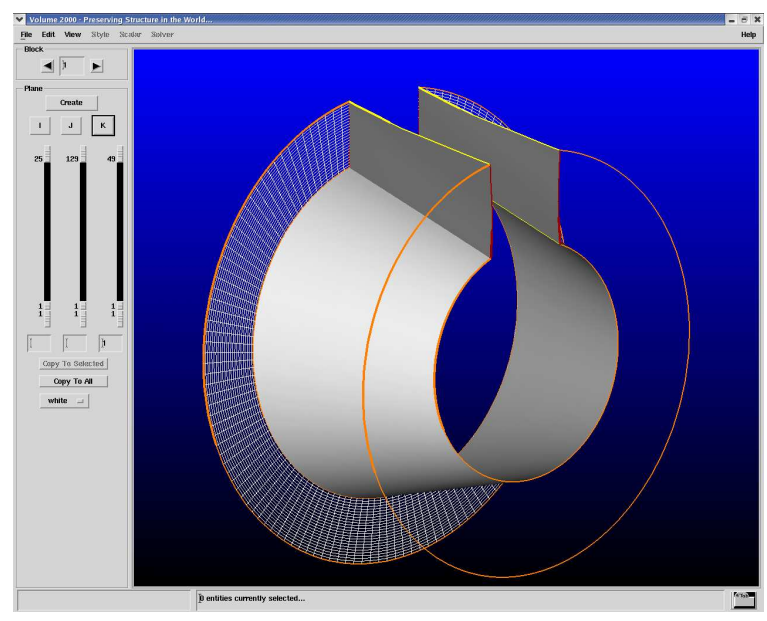

Figure 8. Pseudo-duct geometry

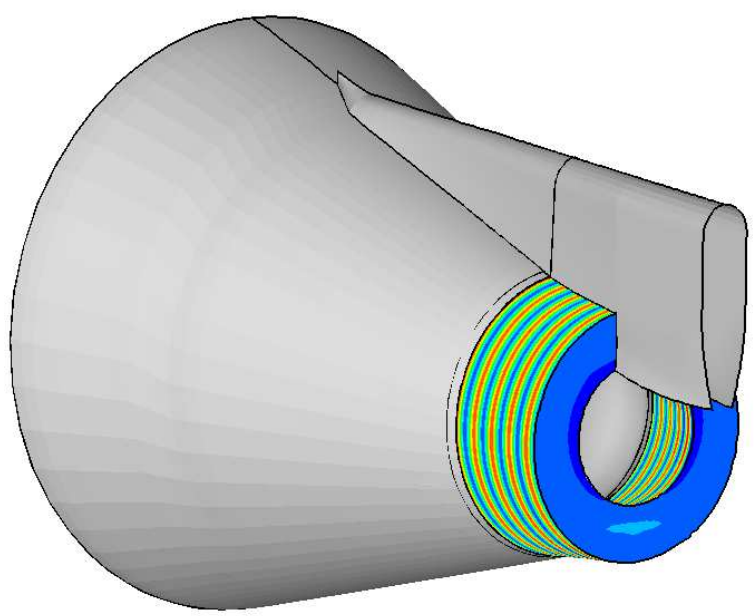

Figure 9. Real Part of Acoustic Potential $\left[M_{1 \_i n i t}=0.7, M_{2}=0.3, \mathbf{f}=1600 \mathrm{~Hz}\right.$, Plane Wave Source]. 
surfaces within the pseudo-duct may then be evaluated by determining RMS pressure at observer locations of interest or overall radiated power.

With this extension in mind, it is useful to summarize the possible steps involved in a full CDUCT-LaRC calculation for the JES geometry in figure 7. With a proper grid and mean flow solutions available from the grid generation and background flow modules, propagation calculations may be performed within the bypass duct. Various liner configurations on any of the surfaces may be included. Results provide acoustic information throughout the bypass duct up to the exhaust plane. At this point, radiation to farfield observers could be computed. However, the acoustic and mean flow data may also be used to perform propagation calculations within a pseudo-duct defined by the core cowl and shear layer surfaces and extending from the exhaust plane to the trailing edge of the core cowl. Radiation calculations may then be performed using the outer surfaces of this pseudo-duct as the source surfaces. This may be done in an effort to include some shear layer refraction effects and/or evaluate the performance of treatment external to the bypass duct but within the pseudo-duct.

\section{Concluding Remarks}

Previous results obtained using CDUCT-LaRC ${ }^{1}$ have shown that the modules for duct propagation and radiation offer appropriate initial models for noise prediction. However, the radiation calculations did not include the aforementioned shear layer boundary condition and instead used the bypass duct exhaust plane as the source surface. In this work, a shear layer boundary condition allowing the extension of CDUCT-LaRC propagation calculations to a pseudo-duct defined by the core cowl and shear layer surfaces has been developed. Initial steps in the implementation have also been discussed. An approximation is proposed whereby the axial wave number for the first radial mode is used in the expression for shear layer impedance for higher order radial modes at the same circumferential order. It is hoped that the importance of higher order will be diminished near the exhaust plane of the bypass duct through attenuation by internal treatment, thereby improving the accuracy of the approximation. An example application has also been presented to illustrate the improvements that it offers for predicting noise radiation from complex inlet and bypass duct geometries. Additionally, the extension provides a framework within which to evaluate treatments in locations within the pseudo-duct, thereby providing a means to evaluate some external treatments in the pursuit of aft-fan noise attenuation. Future work will focus on the application of the shear layer boundary for validation purposes ranging from simplified geometries to complex configurations similar to the example application mentioned previously.

\section{Acknowledgments}

The authors would like to thank Russ Thomas of the Aeroacoustics Branch at NASA Langley and Mike Wiese of the NASA Langley Geometry Laboratory for assistance with the Jet Engine Simulator geometry.

12 of 13

American Institute of Aeronautics and Astronautics 


\section{References}

${ }^{1}$ Nark, D. M., Farassat, F., Pope, D. S., and Vatsa, V., "The Development of the Ducted Fan Noise Propagation and Radiation Code CDUCT-LaRC," AIAA Paper 2003-1652, 2003.

${ }^{2}$ Brentner, K. B. and Farassat, F., "Analytical Comparison of the Acoustic Analogy and Kirchhoff Formulation for Moving Surfaces,” AIAA Journal, Vol. 36, No. 8, 1998, pp. 1379-1386.

${ }^{3}$ Myers, M. K., "On the Acoustic Boundary Condition in the Presence of Flow," Journal of Sound and Vibration, Vol. 71, No. 3, 1980, pp. 429-434.

${ }^{4}$ Farassat, F. and Dunn, M. H., "A Simple Derivation of the Acoustic Boundary Condition in the Presence of Flow," Journal of Sound and Vibration, Vol. 224, No. 2, 1999, pp. 384-386.

${ }^{5}$ Morse, P. M. and Ingard, K. U., Theoretical Acoustics, McGraw-Hill Book Company, 1968.

${ }^{6}$ Eversman, W., "Energy Flow Criteria for Acoustic Propagation in Ducts with Flow," The Journal of the Acoustical Society of America, Vol. 49, No. 6, 1971, pp. 1717-1721. 